\title{
Research technologies for assistance during daily life activities
}

\author{
Marta Gandolla ${ }^{1}$, Alberto Antonietti ${ }^{1}$, Valeria Longatelli ${ }^{1}$, Stefano Dalla \\ Gasperina $^{1}$, Emilia Ambrosini ${ }^{1}$, and Alessandra Pedrocchi ${ }^{1}$ \\ NearLab - Neuroengineering and Medical Robotics Laboratory, Department of \\ Electronics, Information and Bioengineering, Politecnico di Milano, Milano, Italy \\ marta.gandolla@polimi.it
}

\begin{abstract}
An overview of upper limbs exoskeletons aimed at supporting daily life activities of persons with different levels of disabilities is presented, with a particular focus on the functional gain the devices possibly deliver to the user. Observed differences in terms of effective functional improvement and self-perceived functional improvement will be discussed, with particular attention to deriving requirements for a successful assistive device.
\end{abstract}

Keywords: assistive devices (AD), activities of daily living (ADL), functional improvement, externally assessed measurements, self perceived scales

\section{Background}

Severe muscular weakness and chronic disability derived from neuromuscular (e.g., muscular dystrophy (MD), spinal muscular atrophy, spinal cord injuries (SCI) or stroke) or neurodegenerative (i.e., multiple sclerosis (MS), amyotrophic lateral sclerosis) diseases lead to the unavoidable loss of the possibility to perform even simple actions, such as walk, eat, up to change limbs posture. The patients suffer the consequences in terms of independence, quality of life, and self-esteem, given their need to continuously rely on assistance from their caregivers. The development of technologies offers to the patients a way to compensate for their muscular weakness through assistive devices (ADs), which empowers the user in the execution of daily life activities, and are designed to maintain or to improve the functional capabilities of individuals with disabilities. ADs for lower limbs, such as wheelchairs and electric wheelchairs, have been successfully developed and diffused to deal with the deambulation issue. On the other side, the support of upper limbs related activities is more versatile and challenging (e.g., feeding, hygiene, grooming, etc.), and with the increased life expectancy, upper extremity function becomes more and more important to support, especially given the underdevelopment of upper-limbs ADs. Non-ambulant neuromuscular patients identified arm function as their highest priority, including repositioning at night, bring hands to mouth, shift while seated, using the wheelchair joystick and the keyboard of a computer, and personal hygiene as priority functions to be 
regained [9]. The currently existing upper limb assistive devices can be categorized in i) end-effector devices, and ii) exoskeletons. As for end-effector devices, robotic manipulators have as human-machine interface (HMI) point users forearm/hand, and they drive the position of the HMI point. The driving technology can be either passive working through pre-stored mechanical energy - or active working with motors, and able to exert greater forces or control movements more precisely. The main disadvantage of robotic manipulator devices is the impossibility of controlling upper limb joints directly: position changes of the end effector result in unexpected movements of shoulder and elbow joints. As for exoskeletons, they are external electromechanical structures worn by the patient, with joints and links corresponding to those of the human body, in order to work in parallel. These devices can have non-powered using counterweights, springs or elastic bands - or powered controllers, and are usually mounted on a wheelchair. Patients usually prefer exoskeleton as $\mathrm{AD}$, given that these devices help not only to execute the function, but they increase the perception of a self-executed movement. In this work, an extensive literature search has been conducted to identify upper limb ADs, which have been properly tested to assess functional improvement in the execution of daily life activities for neuromuscular patients.

\section{$2 \quad$ Identified Studies}

Studies with participants affected by upper limb disability induced by neurological or neuromotor pathologies were included. The investigated pathologies involve MD, MS, SCI, chronic stroke or in general myopathies that leave patients with muscular weakness. No restrictions have been put in terms of sex and age.

We identified 16 studies, involving 213 subjects. The number of participants in each study ranged from 3 to 55 . All included studies compared patients' ability to perform functional tasks with and without ADs. Most of the studies (11) included at least one subject affected by MD, involving overall $73 \mathrm{MD}$ subjects $[6,15,16,13,4,8,11,17,10,2,5]$, while 3 studies included 33 stroke subjects [12, 7,14 , one study included 3 SCI patients [1], and another study included $4 \mathrm{MS}$ patients [3].

\section{Assistive Devices Used}

All the included studies involved the use of wearable ADs for the upper limb, in particular, seven studies used passive devices [4,6,7,10,13-15], five studies involved active devices $[1,3,5,11,12]$, while other four studies included both active and passive ones $[2,8,16,17]$. ADs used in thirteen studies are available on the market $[2-4,6-8,11-17]$, while the ones involved in the other three are still laboratory prototypes $[1,5,10]$. The passive device that has been employed in most of the studies $[4,6,7,14,15]$ is Wrex, a passive, body-powered antigravity exoskeleton that uses linear elastic bands both for balance and to assist movements in three dimensions against the effect of gravity. In addition to Wrex, 
other passive commercial ADs were Balancer by Focal Meditech [16], Mechanical Top/Help [8]. Another study involved A-gear, a passive arm support still prototype [10]. The active devices, instead, included commercial ADs equipped with at least one actuator, as MyoPro Motion-G by Myomo [12], Armon Products such as the Ayura [11,16], and HapticMaster by Moog FCS Robotics [3]. Two studies used a prototype based on Functional Electrical Stimulation together with an exoskeleton [1] and a completely actuated exoskeleton called BRIDGE/EMPATIA [5].

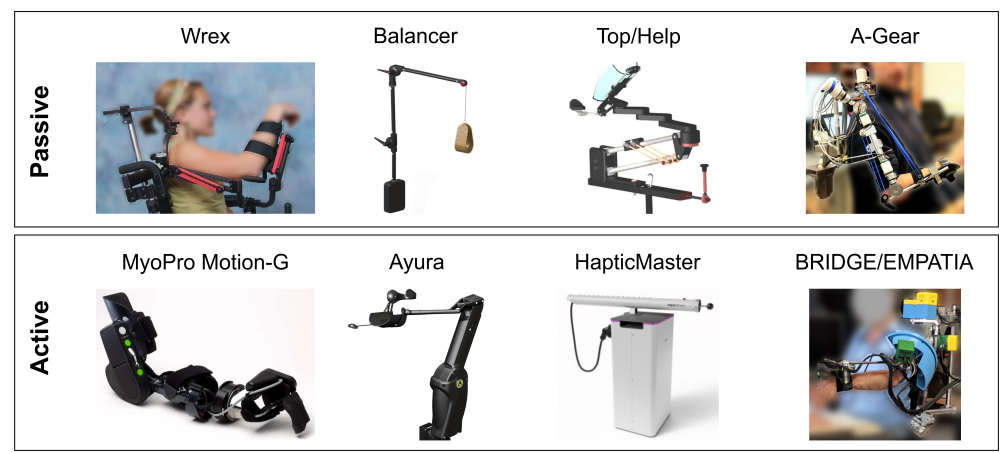

Fig. 1. Different commercial and research upper limb ADs, passive systems in the first row and active ones in the second row

\section{Outcome Measures Analyzed}

The included studies considered different outcome measures, here we have considered only the primary outcome measures. Three studies used the Performance of the Upper Limb scale (PUL), a new tool recently designed for specifically assessing arm functionality in Duchenne MD patients [3,2,5], three studies used the Range Of Motion (ROM) of the shoulder and elbow joint [4, 17, 3], two studies used the upper extremity section of Fugl-Meyer scale [12,14], while the other studies used the Five-point Likert scale [6], the Canadian Occupational Performance Measure [15], the Perceived Functional Benefit [16], the Jebsen Hand Function Test [13], the Fraction Of Reach scale [7], the Action Research Arm Test [8], the Individually Prioritized Problem Assessment [11], and the Root Mean Square Error between the actual and the target angle during elbow flexion and extension [1].

\section{Assistive Device Effects}

All involved ADs improved patients' ability in performing ADL in subjects affected by neuromuscular diseases. We have computed for each study an indicator 
of the $\mathrm{AD}$ efficacy ( $\Delta \mathrm{Perf}$ ), which measured the percentage of functional change with the $\mathrm{AD}$, compared to the baseline condition (without the $\mathrm{AD}$ ). Thanks to these devices, patients increased their actual and perceived capability with the upper limb. In general, the mean improvement was equal to $25.78 \%( \pm 20.13 \%)$. Studies were characterized by different changes, varying from a gain of $5 \%$ to $67.5 \%$. The highest improvements were related to studies that measured the ROM, showing how it really increased thanks to the AD. However, this could implicate an extended working volume, but it was not specifically related to daily life activities. On the contrary, studies characterized by functional outcome measures, such as the PUL, which tested the impact of the AD when performing daily tasks, shown a lower enhancement of the performance indicator.

\begin{tabular}{l|l|l|l|l|l|l|l} 
ID & $\Delta$ Perf & ID & $\Delta$ Perf & ID & $\Delta$ Perf & ID & $\Delta$ Perf \\
\hline$[1]$ & $58.9 \pm 54.1 \%$ & {$[2]$} & $6.5 \pm 17.0 \%$ & {$[3]$} & $65.3 \pm 48.8 \%$ & {$[4]$} & $67.5 \pm 33.4 \%$ \\
{$[5]$} & $15 \pm 15.7 \%$ & {$[6]$} & $12.4 \pm 10.3 \%$ & {$[7]$} & $22.0 \pm 23.8 \%$ & {$[8]$} & $7.7 \pm 21.8 \%$ \\
{$[10]$} & $18 \pm 34.4 \%$ & {$[11]$} & $19.5 \pm 10.8 \%$ & {$[12]$} & $13.2 \pm 10.8 \%$ & {$[13]$} & $10.8 \pm 44.2 \%$ \\
{$[14]$} & $5.0 \pm 22.7 \%$ & {$[15]$} & $37.4 \pm 19.9 \%$ & {$[16]$} & $29.6 \pm 21.7 \%$ & {$[17]$} & $23.8 \pm 46.1 \%$
\end{tabular}

Table 1. Percentage improvement in the performances ( $\Delta$ Perf) measured with the AD with respect to the performances without the $\mathrm{AD}$ (mean \pm standard deviation).

\section{Conclusions}

The present work attempted to investigate the functional effects of upper limbs exoskeletons ADs for neuromuscular patients, both in terms of effectively and perceived regained functionality. The identified studies indicated an important functional gain while using ADs. However, the quality of the included studies was mainly low, given the restricted number of participants involved in the single studies, limitations of participants selection and blindness of outcome assessors, which lead to potentially high risk of bias. On the other hand, all studies were characterized by a positive functional effect across different levels of disability, going towards strong recommendations to use upper limb ADs for neuromuscular patients. The perceived improvements were wider with respect to externally assessed scales, which may indicate a better technology acceptance from the users. In addition, a patient could be motivated and encouraged to use these devices even if the actual gain is perceived only by himself/herself, for example, because he/she can complete the same actions that he/she would complete without support, but with less effort. Future studies may concentrate on the conduction of rigorous trials with the proper number of participants.

Conflict of interest declaration The Authors declare that there is no conflict of interest regarding the publication of this contribution. 


\section{References}

1. Ambrosini, E., et al.: A myocontrolled neuroprosthesis integrated with a passive exoskeleton to support upper limb activities. J.Electromyogr. Kinesiol. 24(2) (2014) 307-317

2. Antonietti, A., et al.: Clinical Benefits and Acceptability of Two Commercial Arm Exoskeletons for Patients with Muscular Dystrophy. In: Converging Clinical and Engineering Research on Neurorehabilitation III. (2019) 31-35

3. Bastiaens, H., et al.: Facilitating robot-assisted training in MS patients with arm paresis: a procedure to individually determine gravity compensation. ICORR 2011 (2011) 5975507

4. Estilow, T., et al.: Use of the Wilmington Robotic Exoskeleton to Improve Upper Extremity Function in Patients With Duchenne Muscular Dystrophy. Am. J. Occup. Ther. 72(2) (2018) 1-5

5. Gandolla, M., et al.: BRIDGE Behavioural reaching interfaces during daily antigravity activities through upper limb exoskeleton: Preliminary results. In: ICORR. (2017) 1007-1012

6. Gunn, M., et al.: User Evaluation of a Dynamic Arm Orthosis for People With Neuromuscular Disorders. IEEE TNSRE 24(12) (2016) 1277-1283

7. Iwamuro, B., et al.: Effect of a gravity-compensating orthosis on reaching after stroke: evaluation of the Therapy Assistant WREX. Arch. Phys. Med. Rehabil. 89(11) (2008) 2121-2128

8. Jan Burgers, M.J.: Upper Limb Training with Dynamic Arm Support in Boys with Duchenne Muscular Dystrophy: A Feasibility Study. Int. J.Phys. Med. Rehab. 03(02) (2015)

9. Janssen, M., et al.: Patterns of decline in upper limb function of boys and men with DMD: an international survey. J.Neurol. 261(7) (2014) 1269-1288

10. Kooren, P., et al.: Design and pilot validation of A-gear: a novel wearable dynamic arm support. J.NeuroEng. Rehab. 12 (2015) 83

11. Lund, K., Brandt, R., Gelderblom, G.J., Herder, J.L.: A user-centered evaluation study of a mobile arm support. In: ICORR, Kyoto, Japan (June 2009) 582-587

12. Peters, H., et al.: Giving Them a Hand: Wearing a Myoelectric Elbow-Wrist-Hand Orthosis Reduces Upper Extremity Impairment in Chronic Stroke. Arch. Phys. Med. Rehabil. 98(9) (2017) 1821-1827

13. Rahman, T., Sample, W., Seliktar, R., Scavina, M.T., Clark, A.L., Moran, K., Alexander, M.A.: Design and testing of a functional arm orthosis in patients with neuromuscular diseases. IEEE transactions on neural systems and rehabilitation engineering: a publication of the IEEE Engineering in Medicine and Biology Society 15(2) (June 2007) 244-251

14. Sanchez, R., Reinkensmeyer, D., Shah, P., Liu, J., Rao, S., Smith, R., Cramer, S., Rahman, T., Bobrow, J.: Monitoring functional arm movement for home-based therapy after stroke. EMBC 7 (2004) 4787-4790

15. Shank: Outcome Measures with COPM of Children using a Wilmington Robotic Exoskeleton. Open J.Occup. Ther. 5(1) (2017)

16. van der Heide, L., de Witte, L.: The perceived functional benefit of dynamic arm supports in daily life. J. Rehab. Res. Dev. 53(6) (2016) 1139-1150

17. Van der Heide, L.A., et al.: Is it possible to assess the effects of dynamic arm supports on upper extremity range of motion during activities of daily living in the domestic setting using a portable motion capturing device? A pilot study. Tech. Disab. 29(1-2) (2017) 91-99 